\title{
SYSTEM OVERVIEW FOR THE MULTI-ELEMENT CORRECTOR MAGNETS AND CONTROLS FOR THE FERMILAB BOOSTER*
}

\author{
C. Drennan", M. Ball, A.R. Franck, D.J. Harding, P.A. Kasley, G. E. Krafczyk, M.J. Kucera, \\ J. Lackey, D. McArthur, J. Misek, W. Pellico. E.J. Prebys, K. Triplett, D. A. Wolff \\ FNAL, Batavia, IL 60510, USA.
}

\begin{abstract}
To better control the beam position, tune, and chromaticity in the Fermilab Booster synchrotron, a new package of six corrector elements has been designed, incorporating both normal and skew orientations of dipole, quadrupole, and sextupole magnets. The devices are under construction and will be installed in 48 locations in the Booster accelerator. Each of these 288 corrector magnets will be individually powered. Each of the magnets will be individually controlled using operator programmed current ramps designed specifically for each type of Booster acceleration cycle. This paper provides an overview of the corrector magnet installation in the accelerator enclosure, power and sensor interconnections, specifications for the switch-mode power supplies, rack and equipment layouts, controls and interlock electronics, and the features of the operator interface for programming the current ramps and adjusting the timing of the system triggers
\end{abstract}

\section{INTRODUCTION}

The goal of the corrector upgrade in the Fermilab Booster is to increase acceleration efficiency in order to meet the needs of the neutrino program through at least the middle of the next decade. The dipole magnets have been designed to provide $1 \mathrm{~cm}$ of beam motion at the highest beam energy $(8 \mathrm{GeV})$, and be able to slew the beam position at least $1 \mathrm{~mm} / \mathrm{ms}$ up through the middle of the acceleration cycle. The normal quadrupoles will allow operators to maintain tune arbitrarily close to upper integer resonance throughout the cycle. The increased distribution of the normal and skew sextupole magnets will allow greater flexibility in controlling beam chromaticity. In order to reduce instabilities produced during beam transition, the slew rates of the quadrupole and sextupole magnets has been increased.

The specification details of the upgraded correctors are found in [1] and [4], and the base design of the new package is introduced in [2].

\section{THE CORRECTOR PACKAGE}

In order to achieve the required strengths for the six magnetic elements in a single, small package, an iron core magnet was designed with twelve poles. The fields are excited by coils around the magnet yoke. Each coil has multiple windings with a variety of numbers of turns and conductor weights. By appropriately connecting the

\footnotetext{
*Work supported by the U.S. Department of Energy
}

\# cdrennan@fnal.gov windings in series across coils, a configuration is produced in which we use a single power supply for each of the six correction elements. The magnet would have been easier to build with twelve simple coils, powered independently to achieve the desired fields. However, complexity in the construction was chosen for the sake of simplicity in operation, removing both the need to recalculate all the power supply ramps when one element is changed and the need to synchronize the power supplies.

Figure 1 is a photo of the corrector package before it is potted.

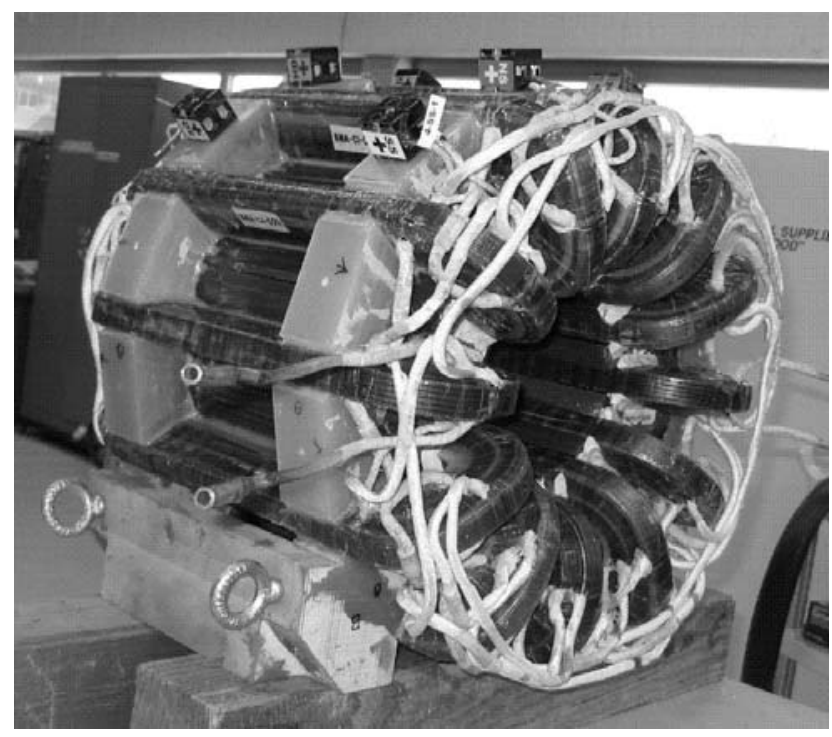

Figure 1 Corrector package before being potted

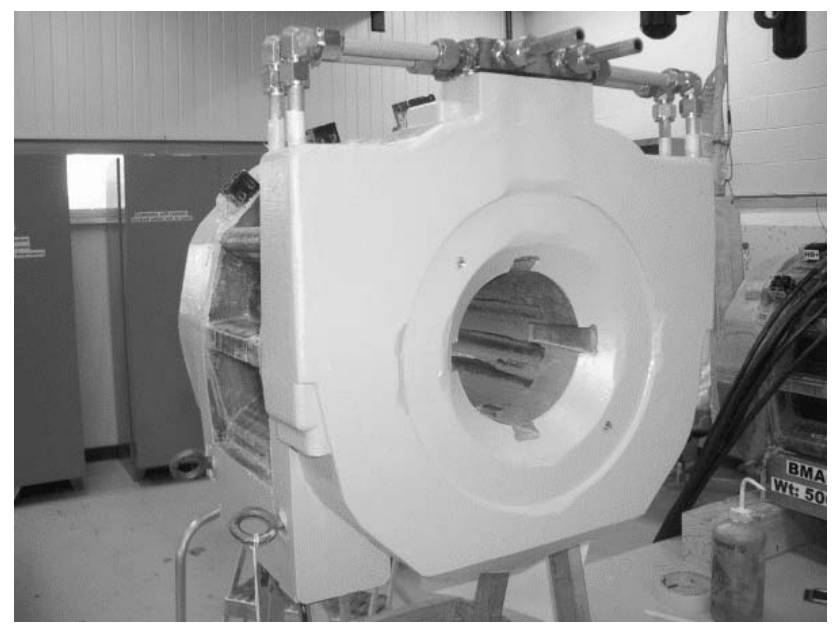

Figure 2 Corrector without BPM after potting and painting. 


\section{PACKAGE INSTALLATION}

Installation of the new correctors will occur in two stages. The installation at the Booster long straight sections will occur during the 2007 shutdown. The long straights are the vertical high beta regions in the machine lattice. Installation in the short straights, the horizontal high beta regions, will occur in 2008 .

Twenty-two of the long straight installations use a common means of supporting the new corrector package. Figure 3 illustrates the standard support which provides for an adjustable stand for making both coarse and fine alignment adjustments.

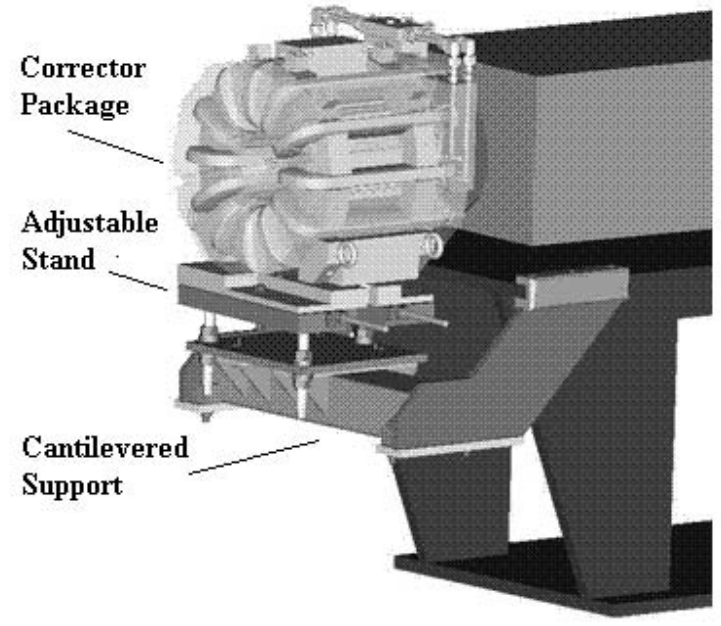

Figure 3 Corrector installation hardware.

\section{THE MAGNET POWER SUPPLIES}

The current sources that will drive the magnet current ramps are four quadrant, DC to DC switching power supplies. These supplies drive the desired current ramp according to a $+/-10$ Volt program reference signal from a ramp controller card. The supplies are similar to the others used throughout the laboratory, but have been designed to provide the increased current and current slew rates required with the new correctors. There are three types of supplies employed in this application. Table 1 lists these types with their output current rating, the number of supplies that will be put into service, and the specification of the power cables used to connect the supplies to the correctors.

\begin{tabular}{|l|l|c|l|}
\hline Type & Qty & Current Rating & Cable to Magnet \\
\hline I & 48 & $65 \mathrm{Amp}$ & 4 Cond. 8 AWG \\
\hline II & 192 & $40 \mathrm{Amp}$ & 2 Cond. 8 AWG \\
\hline III & 48 & $2 \mathrm{Amp}$ & 2 Cond. 12 AWG \\
\hline
\end{tabular}

Fault conditions monitored which will shut the power supplies down include ground fault, RMS overcurrent, DC overcurrent and heat sink over temperature. Each supply has its own external permit control input. The 160 Volt bulk supply can be disabled by Klixon over temperature sensors on each corrector package and safety permits from the accelerator control system.

Diagnostic analog monitor outputs are available for both remote control system monitoring and monitoring at the rack. The monitor outputs include load current and voltage, output RMS current, program reference, and current tracking error.

\section{MAGNET RAMP CONTROLLER CARDS}

A new CAMAC module has been designed for the corrector upgrade [3]. The C473 is a programmable ramp controller capable of generating four semi-independent, time-based analog outputs. These outputs are updated at a $100 \mathrm{kHz}$ rate, and drive the ramp program input of the corrector power supplies.

The C473 also contains digital control capabilities to turn on, turn off, and reset four power supplies. It can also return eight status bits, a ramp enable bit, and a power supply enable bit from each of the power supplies.

The ramp outputs will have the form:

$$
\text { output }=\text { scale_factor } * f(t)+\text { offset }
$$

where:

- $\quad$ scale_factor is a constant scale factor having a range of -128.0 to +127.9

- $\mathrm{f}(\mathrm{t})$ is an interpolated function of time which is initiated by a TCLK event. $f(t)$ defines the overall shape of the output function

- offset is a constant offset having a range of -32768 to +32767

The output functions of all four channels share a common TCLK trigger. Each channel's output function will then start playing out after an independent delay, programmable from 30 to $255 \mu$ sec. Different TCLK triggers also signal different Booster acceleration modes. The scale factor and the offset can be selected independently for each Booster acceleration mode, for each channel.

The outputs are provided in analog format (16 bits, +/$10.000 \mathrm{~V})$.

Each channel's final output is constantly compared to a voltage feedback signal from that channel's power supply. The difference between output and feedback is monitored by the FPGA through an ADC and compared to a programmable error threshold value. In the event of a violation of the error threshold, a status bit and/or CAMAC Look-At-Me (LAM) can be set.

\section{MONITORING ANALOG SIGNALS}

All five analog monitor outputs of each power supply and three monitor outputs from each bulk supply are available for monitoring and logging on the main accelerator control system. There will be 256 channels of multiplexed analog digitization at each of the six equipment rack locations. This is implemented using two 128 channel Hotlink Rack Monitor (HRM) chassis at each 
location. These HRM's stream the digitized data to MVME processor modules. One at each location. These processors parse the streams of data and serve this data to the control system as requested.

\section{THE EQUPMENT RACKS}

The 288 power supplies and associated controller cards and control system equipment for monitoring output readbacks are assembled into four racks at each of six locations around the Booster Gallery. Each location will supply power for eight of the six element corrector packages, four in long straight areas and four in short straight areas. Only two of the four racks in each area will be populated in 2007. These will support the long straight correctors. Figure 2 illustrates the layout of these racks.

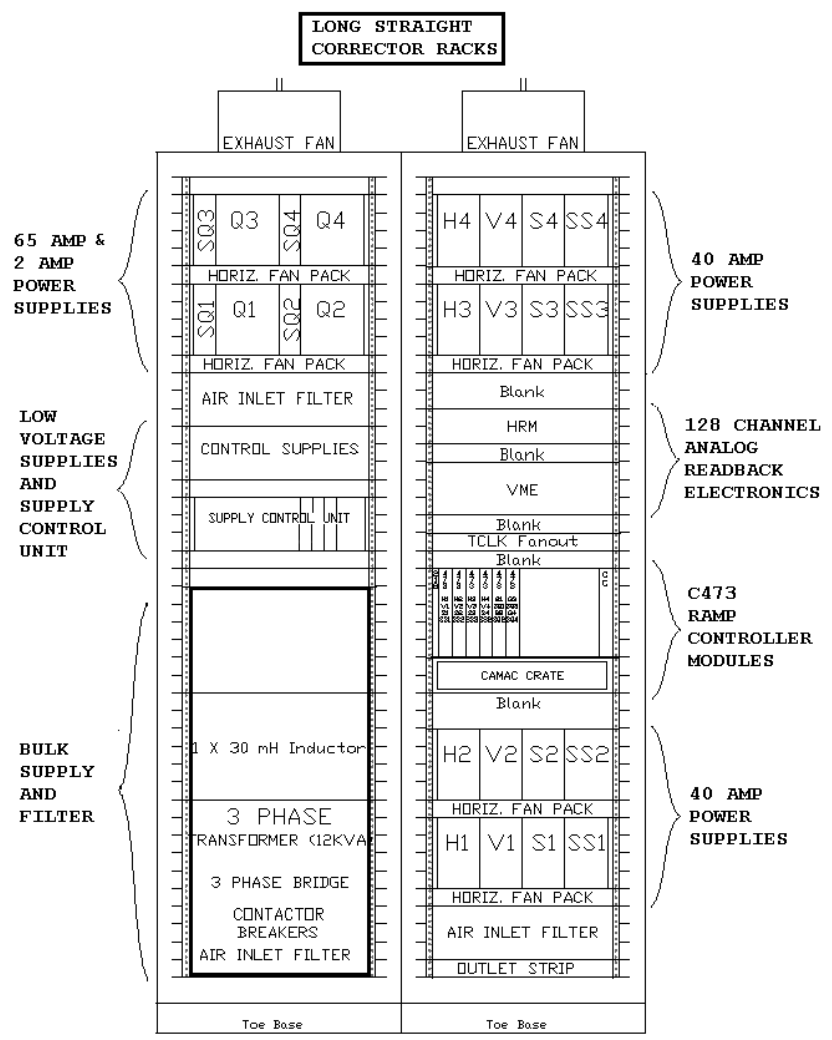

Figure 3 Layout of Long Straight Corrector Racks

\section{CONTROL SYSTEM APPLICATIONS}

Several computer control applications that are related to the operation of the current set of corrector magnets are being adapted to the new corrector system. There are applications which can directly manipulate and monitor individual power supplies. There are programs for creating, loading, and managing and archiving the various ramp curves used for the various corrector elements and various Booster cycles modes. There are higher level programs that allow operators to set up three bumps, a means of managing beam positions in different places around the ring. There are also programs for automated orbit correction and tune correction.

\section{CONCLUSIONS}

The new multi-element corrector packages and associated power supplies and controls will increase the control operators have in determining beam position, tune and chromaticity of the Booster beam throughout the acceleration cycle. This will allow more beam to be accelerated and delivered to the experiments while keeping the beam losses low and activation levels in the Booster enclosure below safety limits.

\section{REFERENCES}

[1] E.J. Prebys, et al., "Booster Corrector System Specification", Fermilab Beams-doc-1881-v5, 2006, http://beamdocs.fnal.gov/.

[2] V.S. Kashikhin et al., "A New Correction MagnetPackage for the Fermilab Booster Synchrotron" in Proc. of 2005 PAC, Knoxville, Tennessee, pp. 1204-1206.

[3] D. McArthur, " CAMAC 473 Quad Ramp Controller", Fermilab Beams-doc-2361-v3, 2007, http://beamdocs.fnal.gov/.

[4] E.J. Prebys, et al., "New Corrector System for the Fermilab Booster", MOPAS016, PAC '07 (2007) 